\title{
Novel approach on the synthesis of starch betainate by
}

\section{transesterification}

Mohit Sharma a ${ }^{\text {a }}$ Roberto Aguado ${ }^{\text {b, }}$, Dina Murtinho ${ }^{\text {b }}$, Artur J. M. Valente ${ }^{\mathrm{b}}$, Paulo J. T. Ferreira ${ }^{\mathrm{a}}$

${ }^{\text {a }}$ University of Coimbra, CIEPQPF, Department of Chemical Engineering, Rua Sílvio Lima, Pólo II

- Pinhal de Marrocos, 3030-790 Coimbra, Portugal

${ }^{\mathrm{b}}$ University of Coimbra, CQC, Department of Chemistry, Rua Larga, 3004-535 Coimbra, Portugal

* rag@uc.pt (Roberto Aguado)

Código de campo alterado

Non-standard abbreviations:

Formatada: Inglês (Reino Unido)

BetHCl: betaine hydrochloride

BM: ball milling

CS: cationic starch

MeBetCl: methyl betainate chloride

SB: starch betainate

$\mathrm{SB}_{\mathrm{D}}$ : starch betainate, dry method

$\mathrm{SB}_{\mathrm{S}}$ : starch betainate synthesised in solution 


\section{Abstract}

Transesterification of starch with methyl betainate was studied for the first time, both in aprotic media and in solid state, and both under alkaline and acidic conditions. Betaine hydrochloride was first esterified in methanol, attaining a conversion of $86 \%$. Starch was then converted into starch betainate in either $N, N$-dimethylformamide or dimethyl sulfoxide, and using sulfuric acid as catalyst or pre-activating the polymer in $\mathrm{NaOH} /$ ethanol. Furthermore, solid-state transesterification was carried out in a ball mill, for which sulfuric acid was replaced with the less corrosive sulfamic acid. Cationic starch esters were characterised by ${ }^{1} \mathrm{H}$ and ${ }^{13} \mathrm{C}$ NMR spectroscopy, infrared spectroscopy, thermogravimetric analysis, viscometry, optical microscopy (in water) and scanning electron microscopy (dry). In solution, the process attained degrees of substitution up to 0.4. No by-products, dehydration, oxidation or colouring were detected, but starch underwent severe depolymerization in wet media. In solid state, whilst the resulting degree of substitution was lower, degradation was minimal. In any case, transesterification, with its variety of possibilities, yields cationic starches that offer a promising alternative to conventional ethers.

\section{Keywords}

Betaine methyl ester, Starch betainate, Transesterification

\section{Introduction}

Conventional, commercially available cationic starches (CSs) are produced by etherification of starch with either (3-chloro-2-hydroxypropyl)trimethylammonium chloride (CHPTAC) or (2,3epoxypropyl)trimethylammonium chloride (EPTAC) [1]. These CSs are extensively used in papermaking, both as wet end additive and as sizing agent [2-4]. Moreover, they have been proven to be effective flocculants for wastewater treatment [5]. Due to their alleged biodegradability, along 
with the high availability of starch, CSs are frequently advocated as environmentally-friendly alternatives to synthetic polyelectrolytes [2,6]. However, some issues have put the greenness of cationic polysaccharide ethers under question [7]. EPTAC (or CHPTAC) ultimately comes from propylene, the reaction produces undesirable by-products, and highly substituted CS ethers actually show poor biodegradability [8].

Due to the aforementioned concerns, the use of biobased reagents for starch modification has been an aspect of major interest for several industries $[9,10]$. The replacement of etherifying cationizing reagents by a naturally found amino acid, betaine hydrochloride, leads to nontoxic and more biodegradable CS esters than their ether counterparts [11-14]. CS esters, containing more labile cationic groups, were first proposed in 2000 , standing as a promising dry strength additive to be used at the wet end of the paper machine [12]. Betaine was converted into its acyl chloride and subsequently reacted with starch, in the presence of pyridine. However, undesired colouring was reported for starch betainate (SB) with high degrees of substitution (DS) [12]. Nonetheless, the chlorination step is not mandatory, as some researchers have reported the production of starch betainate by Steglich esterification, using $N, N^{\prime}$-diisopropylcarbodiimide and 4dimethylaminopyridine [11]. In this case, the medium was 1,4-dioxane, in which neither native or cationic starch is soluble, and DS below 0.3 were obtained. Recent advances in the synthesis of SB are scarce, as CS ethers are still clearly predominant [2], but the valorisation of betaine keeps being the source of much research $[15,16]$.

In the present paper, transesterification is proposed as a novel alternative synthetic route to produce SB. At the best of our knowledge, transesterification of starch has been carried out with a small number of esters, including vinyl laureate [17] and vinyl acetate [18], and using $\mathrm{K}_{2} \mathrm{CO}_{3}$ as base catalyst. The reaction of polysaccharides with fatty acid methyl esters was also studied using organic catalysts such as triazabicyclodecene, or alternative reaction media like ionic liquids $[19,20]$. 
While the modification of starch in water or organic solvents is prevalent in the literature,

commercial CS ethers are frequently obtained by semi-dry or dry methods [2]. Ball milling (BM) is a dry state, cost effective and eco-friendly approach, which uses mechanical actions such as friction, collision, impingement and shear between grinding balls and container walls [21,22]. Some key physicochemical properties of starch get clearly affected by BM. Its crystallinity decreases, becoming more reactive [23,24]. Its porosity increases by developing cavities in the granules [25], and so does the pasting stability, resulting in lower viscosity when dispersed in water [26]. Starch nanoparticles in the range of 9-12 $\mathrm{nm}$ can be obtained from 1-100 $\mu \mathrm{m}$ granules [27]. However, none of those contributions implied a chemical reaction concurrently with BM. In this regard, Su et al.'s [28] and Li et al.’s [29] dry synthesis of cationic starch ether and octenyl succinate starch, respectively, should be mentioned. The authors highlighted that the structural disruption of starch granules by BM increased the reactivity.

We report for the first time the synthesis of starch betainate by transesterification, and also the use of a ball mill reactor for hosting starch cationization. Methyl betainate was synthesised and transesterified with corn starch, both in homogenous conditions (wet method) and concurrently with ball milling. The influence of the betaine-to-starch ratio, the choice of either base catalyst or acid catalyst, and the importance of a previous enzymatic hydrolysis were explored.

\section{Material and Methods}

\subsection{Materials}

Corn starch (27.3\% amylose) of industrial origin was dried at $100{ }^{\circ} \mathrm{C}$ for $2 \mathrm{~h}$ before use. Its average molecular weight, as estimated by viscometry (ISO 3105) and using Mark-Houwink parameters [30], is ca. $3.2 \times 10^{5} \mathrm{~g} \mathrm{~mol}^{-1}$, corresponding approximately to a degree of polymerization (DP) of $2.0 \times 10^{3}$. 
Betaine hydrochloride (BetHCl, 99\%) was purchased from Alfa Aesar and used as received, for esterification. Thionyl chloride (97\%) was obtained from Sigma-Aldrich. Sulfamic acid (99.3\%) was bought from Fluka Analytical. All solvents were purified or dried prior to use following standard procedures. Other commercially available compounds were used without further purification.

\subsection{Synthesis of starch betainate}

The synthesis of SB was carried out in two steps: (i) synthesis of the intermediate compound (betaine methyl ester chloride, $\mathrm{MeBetCl}$ ) through esterification of betaine hydrochloride, and (ii) synthesis of SB by transesterification. This transesterification was carried out in a polar aprotic solvent (in solution) and in a ball mill (solvent-free), as schematically depicted in Fig. 1.

\subsubsection{Intermediate synthesis}

The synthesis of $\mathrm{MeBetCl}$ was carried out using $\mathrm{SOCl}_{2}$ and methanol, following a previously described method for the esterification of glycine [31]. The process reported here, however, aimed at maximum efficiency by using a higher amino acid concentration. Briefly, $4.72 \mathrm{~mL}$ of thionyl chloride $(65.1 \mathrm{mmol})$ were added dropwise to $50 \mathrm{~mL}$ of methanol in an ice bath, followed by addition of $10.0 \mathrm{~g}$ of $\mathrm{BetHCl}(65.1 \mathrm{mmol})$. The resulting mixture was then refluxed for $4 \mathrm{~h}$ at $70{ }^{\circ} \mathrm{C}$. Afterwards, the solvent was removed by using a rotary evaporator, giving semi-crude $\mathrm{MeBetCl}$, which was triturated twice with diethyl ether to remove the excess of dimethyl sulfite. MeBetCl was vacuum-dried and repeatedly washed with diethyl ether, until no yellow colour remained.

\subsubsection{Transesterification in solution}

The transesterification was performed with the use of native or cooked starch and $\mathrm{MeBetCl}$ in alkaline/acidic media. Cooking, whose main purpose was the disruption of the crystalline or semicrystalline structure of amylose and amylopectin, is described in the Supplementary Information. 
The alkaline medium was not attained with the addition of any base catalyst to the reaction mixture. Instead, native and cooked starch were pre-activated by refluxing with a boiling $\mathrm{NaOH}$ ethanolic solution $(8 \mathrm{w} / \mathrm{w} \%$ ) for $60 \mathrm{~min}$. Then, the alkali starch suspension was vacuum filtered, rinsed with ethanol to remove excess $\mathrm{NaOH}$ and washed with diethyl ether to remove the ethanol completely. Activated starch was stored under vacuum for one day at most or used immediately in the transesterification reactions. $0.49 \mathrm{~g}$ of alkali native/cooked starch, corresponding to $3 \mathrm{mmol}$ of anhydroglucose units (AGU), and $0.50 \mathrm{~g}$ of $\mathrm{MeBetCl}(3 \mathrm{mmol})$ were dissolved in $10 \mathrm{~mL}$ of DMF or DMSO and kept under reflux for $24 \mathrm{~h}$ at $70{ }^{\circ} \mathrm{C}$. The same reaction was also performed using 1:2 and 1:3 molar ratios of alkali native/cooked starch to MeBetCl.

For the acid-catalyzed transesterification, the reaction mixture containing native/cooked starch $(0.49$ g, $3 \mathrm{mmol})$ and $\mathrm{MeBetCl}(0.50 \mathrm{~g}, 3 \mathrm{mmol})$ was dissolved in a $0.04 \mathrm{M} \mathrm{H}_{2} \mathrm{SO}_{4}$ solution of DMF or DMSO $(10 \mathrm{~mL})$ and kept under reflux for $24 \mathrm{~h}$ at $70{ }^{\circ} \mathrm{C}$. The same reaction was also performed using 1:2 and 1:3 molar ratios of native/cooked starch to MeBetCl.

In both cases, SB was precipitated using absolute ethanol, vacuum filtered and stored in an oven at $50{ }^{\circ} \mathrm{C}$. The final product from the transesterification in solution is mentioned as $\mathrm{SB}_{\mathrm{S}}$ (starch betainate produced in solution) in further discussion.

\subsubsection{Solvent-free transesterification}

The transesterification reaction was also performed in a ball mill (BM; Retsch, model MM200) using native/cooked corn starch and $\mathrm{MeBetCl}$, both under alkaline and acidic conditions, and without the addition of any liquid substance. Ball milling ensured the homogenization of the reaction mixture and promoted the collision between the reactant particles. The grinding jar (stainless steel, $10 \mathrm{~mL}$ ) contained two steel balls with a diameter of $5 \mathrm{~mm}$, the shaking frequency was set at $25 \mathrm{~s}^{-1}$ and the reaction time was $2 \mathrm{~h}$. 
For the base-catalysed transesterification, the reaction was performed using $\mathrm{MeBetCl}(0.50 \mathrm{~g}, 3$ $\mathrm{mmol})$ and alkali cooked starch $(0.49 \mathrm{~g}, 3 \mathrm{mmol})$, obtained in $\mathrm{NaOH} /$ ethanol as aforementioned, in the grinding jar of the BM. Regarding the acid-catalysed process, $0.01 \mathrm{~g}$ of sulfamic acid were added to the same amounts of $\mathrm{MeBetCl}$ and cooked starch. Solvent-free transesterification of both types (base- and acid-catalysed) was also performed for a 1:2 molar ratio of AGU to MeBetCl.

The resulting dry mixture was collected and dissolved in distilled water with stirring and heating, followed by precipitation from ethanol, in order to remove remaining $\mathrm{MeBetCl}$ and, where applicable, the sulfamic acid. The final product from this process is mentioned as $\mathrm{SB}_{\mathrm{D}}$ (starch betainate produced by the dry method) from now on.

\subsection{Characterization of starch betainate and intermediate products}

Attenuated Total Reflectance-Fourier-transform infrared (ATR-FTIR) spectra were recorded by

using an Agilent Cary 630 device, from 750 to $3000 \mathrm{~cm}^{-1}$, at a resolution of $4 \mathrm{~cm}^{-1}$ and 64 scans per sample. Nuclear magnetic resonance (NMR) spectra were obtained from a Bruker Biospin GmbH spectrometer, at $400 \mathrm{MHz}$ for ${ }^{1} \mathrm{H}-\mathrm{NMR}$ and at $100 \mathrm{MHz}$ for ${ }^{13} \mathrm{C}-\mathrm{NMR}$, using $\mathrm{D}_{2} \mathrm{O}$ or DMSO-d 6 (Eurisotop, 99.8\%) as solvent and 3-(trimethylsilyl) propionic-2,2,3,3-d4 acid sodium salt (Eurisotop, 98\%) as internal reference. Few $\mu \mathrm{L}$ of $\mathrm{NaOD}$ (40\%, Eurisotop) were also added in some SB samples to dissolve them quickly in $\mathrm{D}_{2} \mathrm{O}$. The degree of substitution was calculated from the area of the resonance assigned to the methyl protons of the quaternary ammonium group.

Thermogravimetric analysis (TGA) was carried out on a thermo-microbalance TG 209 F3 Tarsus, from Netzsch Instruments. Samples were heated from $40{ }^{\circ} \mathrm{C}$ to $600{ }^{\circ} \mathrm{C}$, under flow of nitrogen $(20$ $\mathrm{mL} \min ^{-1}$ ) and at a rate of $10{ }^{\circ} \mathrm{C} \min ^{-1}$. Kinematic viscosity was determined using a size 100 Cannon-Fenske viscometer in a thermostatic bath (TAMSON TV 2000) set at $40^{\circ} \mathrm{C}$. Measurements followed the ISO 3105 standard. Polymer solutions were prepared with a concentration of $5 \mathrm{mg}$ 
$\mathrm{cm}^{-3}$ in DMSO. The surface morphology of starch granules, precipitated cooked starch, conventional $\mathrm{CS}, \mathrm{SB}_{\mathrm{S}}$ and $\mathrm{SB}_{\mathrm{D}}$ were observed by scanning electron microscopy (SEM). Backscattered-electron imaging was performed by means of a tabletop microscope TM4000 Plus from Hitachi.

\section{Results and discussion}

\subsection{Intermediate synthesis}

The addition of $\mathrm{SOCl}_{2}$ to methanol generated methyl chloride, which reacted with $\mathrm{BetHCl}$ in a nucleophilic substitution, as depicted in Scheme 1. Grinding the precipitate led to recover $1.054 \mathrm{~g}$ of dry impure crystals per gram of BetHCl used. The conversion into $\mathrm{MeBetCl}$ was as high as $86 \%$, calculated from the ${ }^{1} \mathrm{H}$ NMR spectrum shown in Fig. 2a.

The singlets at $3.34,3.83$ and $4.36 \mathrm{ppm}$ correspond to the nine hydrogens of the quaternary ammonium group of $\mathrm{MeBetCl}$ and $\mathrm{BetHCl}$ the three hydrogens of the methyl ester and the two methylene hydrogens of $\mathrm{MeBetCl}$, respectively (Fig. 2a). The singlet at $4.31 \mathrm{ppm}$ is due to unconverted BetHCl. The formation of the ester bond is also supported by the ${ }^{13} \mathrm{C}$ NMR spectrum $\left(\mathrm{D}_{2} \mathrm{O}, 100 \mathrm{MHz}\right): \delta(\mathrm{ppm})=54.1,63.4,165.7$ (Fig. S1, Supplementary Information). Esterification leads to an upfield displacement (165.7 ppm) of the signal due to carboxyl groups (166.7 ppm).

In Fig. 3a, the FTIR spectrum of MeBetCl is compared to that of BetHCl for the evaluation of structural changes. The characteristic peaks at $1723 \mathrm{~cm}^{-1}, 2974 \mathrm{~cm}^{-1}, 1200 \mathrm{~cm}^{-1}, 1247 \mathrm{~cm}^{-1}, 1473$ $\mathrm{cm}^{-1}$ and $3400-2400 \mathrm{~cm}^{-1}$ can be attributed to $\mathrm{C}=\mathrm{O}, \mathrm{C}-\mathrm{H}, \mathrm{C}-\mathrm{C}, \mathrm{C}-\mathrm{O},-\mathrm{N}\left(\mathrm{CH}_{3}\right)_{3}$ and $\mathrm{O}-\mathrm{H}$ stretching, respectively. In comparison to $\mathrm{BetHCl}$, the two most characteristic features in the spectrum of $\mathrm{MeBetCl}$ are the strong $\mathrm{C}=\mathrm{O}$ band (ester bond), which appears at $1748 \mathrm{~cm}^{-1}$ and is clearly different from the one associated to the carboxyl group, and $\mathrm{C}-\mathrm{O}$ stretching, which appears at $1256 \mathrm{~cm}^{-1}$. 


\subsection{Transesterification in solution}

The type of starch used in each experiment (native or cooked), the molar ratio of MeBetCl to AGU, the type of reaction medium (alkaline or acidic) and the DS are displayed in Table 1. DS values up to 0.4 were calculated for SB synthesised through the use of alkali-activated cooked starch and DMF as solvent (Experiments 1-3). Transesterifications with alkali starches are labelled as "Reaction B" in Scheme 2. In contrast, with the same solvent, less, substitution was obtained in the presence of an acid catalyst (Experiments 5-8, Scheme 2, reaction C). Since all reactions in solution took place for $24 \mathrm{~h}$, we can safely assume that the DS attained in each case is determined by equilibrium.

Clearly, the use of starch in its cooked form achieved higher, substitution, which can be explained by its lower degree of molecular order (less crystallinity) and its higher solubility. In other words, and once again in terms of chemical equilibrium, cooking favoured the consumption of reagents (Scheme 2) by irreversibly changing key thermodynamic properties, namely the entropy of the polymer.

The poor efficiency of reaction in DMSO, especially with alkali starch (Experiments 9-12) wwas unexpected, as this solvent has been proven suitable for the esterification of starch $[17,18]$. The lower DS values were likely due to degradation in these particular media [32,33]. Hence, a large part of the product was lost as cationic maltodextrins or other oligosaccharides that could not be recovered by precipitation. Since native starch was more resistant to this degradation, experiment 12

(Table 1) was the only case in which the DS achieved surpassed that of cooked starch.

\subsubsection{Insights into molecular structure}

Fig. 2c shows the ${ }^{1} \mathrm{H}$ NMR spectrum for a representative SB sample, obtained in DMF using alkali

Eliminado: 4

Eliminado: II

High

Eliminado: of $0.16,0.33$ and 0.4

Eliminado: depicted

Eliminado: If the same experiments were carried out in the presence of native/cooked starch, acid catalyst, DMF as a solven (Experiments 5-8)

Eliminado: lower

Eliminado: DS were

Eliminado:

Eliminado: were carried out

Eliminado: The larger the excess of $\mathrm{MeBetCl}$, the larger the conversion. Also, alkaline activation seemed to favor the reaction, as it resulted in higher DS than the acid-catalysed process. Furthermore,

Eliminado: increased

Eliminado: the DS achieved

Eliminado: efficiency of reaction was found to be

Eliminado:

Eliminado: This

Eliminado: allowed good results in

Eliminado: , whilst only the least soluble, least substituted fraction could be analysed

Eliminado: Interestingly enough, and unlike aforementioned for DMF, reactions in $\mathrm{DMSO} / \mathrm{H}_{2} \mathrm{SO}_{4}$ outperformed those in DMSO (Experiments 14 and 15). 
methyl groups of the quaternary ammonium. The resonances from 3.5 to $4 \mathrm{ppm}$ represent the hydrogens attached to carbons 2, 4, 5, 6 (H-6 and H-6'), and 3 of AGU, typically in that order. The doublet for the H-1 $(\alpha)$ anomeric proton lies downfield (5.35 ppm). There was a certain shift upfield of all signals upon cationization, but the clearest change among AGU protons was the signal broadening of $\mathrm{H}-6$ and $\mathrm{H}-6$ ',

The ${ }^{1} \mathrm{H}-\mathrm{NMR}$ spectra of samples from other reaction conditions are qualitatively identical, differing only in the peak areas, as can be seen from data shown in Fig. S1 (Supplementary Information). This also contains the ${ }^{13} \mathrm{C}-\mathrm{NMR}$ spectrum of SB obtained in acidic medium, Experiment $6(100 \mathrm{MHz}$, $\left.\mathrm{D}_{2} \mathrm{O}\right): \delta(\mathrm{ppm})=53.3$ (quaternary ammonium methyl groups), $60.4(\mathrm{C}-6), 66.1$ (betainate's methylene), 69.3-73.3 (C-2, C-3, C-5), 76.9 (C-4), $99.7(\mathrm{C}-1), 169.2(\mathrm{C}=\mathrm{O})$ [34]. In comparison, the ${ }^{13} \mathrm{C}-\mathrm{NMR}$ spectrum of an alkaline sample (Experiment 2) is similar, with the exception of the C2/C-3/C-5 collective signals. They are shifted downfield, accounting for an average $\Delta \delta$ of $+2.2 \mathrm{ppm}$ from the spectrum of unmodified starch. In both cases, the $\Delta \delta$ of C- 6 is $+2.9 \mathrm{ppm}$, evidencing substitution on the primary hydroxyl group (OH-6). This was clearly preferential under acid catalysis, while alkaline pre-activation led to a more evenly distributed substitution on the $-\mathrm{OH}$ groups of AGU.

All mid-IR spectra in Fig. 3b display some typical fundamental bands of polysaccharides at 3273 $\mathrm{cm}^{-1}$ and $2912 \mathrm{~cm}^{-1}$, related to the O-H and C-H stretching vibration, respectively. Bands at 1341 $\mathrm{cm}^{-1}, 1149 \mathrm{~cm}^{-1}, 994 \mathrm{~cm}^{-1}$ and $860 \mathrm{~cm}^{-1}$ indicate $\mathrm{C}-\mathrm{H}$ stretching, $\mathrm{C}-\mathrm{O}-\mathrm{C}$ asymmetrical stretching, $\mathrm{C}-\mathrm{O}-\mathrm{H}$ and $\mathrm{C}-\mathrm{O}$ stretching vibrations, respectively. The band at $927 \mathrm{~cm}^{-1}$ is associated with Dglucopyranose ring vibration. The strong absorption band at $1022 \mathrm{~cm}^{-1}$ can be attributed to $\mathrm{C}-\mathrm{O}-\mathrm{H}$ stretching, while that at $1242 \mathrm{~cm}^{-1}$ is specific to carbon 6 of AGU [35]. The ATR-FTIR spectrum of alkali starch is also provided in Fig. $3 \mathrm{~b}$ to prove the success of deprotonation: the prominent peak at $1428 \mathrm{~cm}^{-1}$ (HCO in-plane bending) matches the spectra recorded for what other authors call "caustic starch" [36]. After transesterification and washing, all $\mathrm{C}-\mathrm{O}^{-} \mathrm{Na}^{+}$have been either substituted or
Eliminado: , given the coexistence of substituted and unsubstituted units. This affected more clearly the two C-6 hydrogens

Eliminado: file

Eliminado: qualitatively identical

Eliminado: 5

Eliminado: The absorption at $1648 \mathrm{~cm}^{-1}$ is attributed to an $\mathrm{O}-\mathrm{H}$ bending vibration due to the sorption of water. Other $b$

Eliminado: such as those

Eliminado: ,

Eliminado: the

Eliminado:

Eliminado: $\mathbb{q}$

Eliminado: 5 
regenerated, and new peaks at $1458 \mathrm{~cm}^{-1}$ and $1750 \mathrm{~cm}^{-1}$ can be observed owing to quaternary ammonium groups and ester bonds, respectively [37,38]. It should be noted that when sulfuric acid is used, the ester band is shifted to $1740 \mathrm{~cm}^{-1}$. Moreover, a barely noticeable band at $2972 \mathrm{~cm}^{-1}$ can be assigned to methylene $\mathrm{C}-\mathrm{H}$ stretching in betainate moieties.

\subsubsection{Thermal stability}

Thermal degradation and differential thermogravimetry curves of native corn starch, cooked starch, starch betainates and a cationic starch ether prepared conventionally with CHPTAC [5] are shown in Fig. 4. It should be noted that these cationic starches were synthesised from the same cooked starch.From Fig. 4a, it can be observed that the cooking of starch, involving hydrolysis and disruption of crystalline or semicrystalline domains, had a major impact on the degradation profile. As the molecular disorder increases, the temperature corresponding to the maximum degradation rate, or $T_{\max }$ (Fig. $\underline{4 \mathrm{~b}}$ ), is shifted from $304{ }^{\circ} \mathrm{C}$ to $239^{\circ} \mathrm{C}$. Moreover, the separation of amylose and amylopectin, from being packed together in pristine starch granules [2], gives rise to two transitions, the first one being associated with amylopectin and the second one with amylose. This distinction vaguely remains in the $\mathrm{SB}_{\mathrm{S}}$ obtained by alkaline transesterification, whose thermal stability is slightly higher $\left(T_{\max }=247^{\circ} \mathrm{C}\right)$.

Interestingly enough, the thermal behaviour of the CS ether was closer to that of native starch, regaining some of the thermal stability that was lost by cooking. The key difference does not lie in the kind of functional group (ester/ether), given that the acid-catalysed transesterification resulted in a SBS with $T_{\max }=287^{\circ} \mathrm{C}_{\mathrm{v}}$ It can be suggested that alkali and acid had opposite effects on molecular packing: the former prevented the formation of intra- and intermolecular hydrogen bonds, and the latter depolymerised selectively the less ordered domains to sugars that were lost upon filtration.
Eliminado: $\mathbb{1}$

The spectra recorded for other starch betainates were qualitatively similar.

Eliminado: obtained by base- and acid-catalyzed transesterification (MeBetCl/AGU = 2)

Eliminado:

Eliminado: (DS $=0.3$ )

Eliminado: 6

Eliminado: $\mathbb{T}$

Eliminado: $6 \mathrm{a}$ 
This way, the degree of molecular order of the product became higher than that of the starting cooked starch.

\subsection{Solvent-free transesterification (dry method)}

Both in solution and in solvent-free processes, either an acid or a base catalyst was required to detect the presence of SB by the techniques aforementioned ( ${ }^{1} \mathrm{H}$ NMR, ATR-FTIR). The choice of sulfamic acid for solid state, acid-catalysed transesterification, instead of sulfuric acid, was due to its high melting point $\left(205^{\circ} \mathrm{C}\right)$, its lack of hygroscopicity and its lower corrosivity on steel surfaces. Despite its reactivity, no by-products were detected by analytical techniques after washing.

Regardless the reaction B and C (Scheme 2) taking place in solid state or in solution, the mechanism is the same: a reversible bimolecular substitution. Even in the absence of solvents and without heating, collision and high shear forces between the grinding balls and the container walls allowed for the reaction to occur [28]. However, since the BM was stopped after only 2 hours, equilibrium values were not reached. The type of catalyst, acid or alkaline pre-activation, showed no effect on DS. Like in the case of the wet method, the reaction proceeded to a greater extent when the molar ratio of $\mathrm{MeBetCl}$ to $\mathrm{AGU}$ was higher (Table 2). While these DS values are much lower than those obtained with DMF, still a DS of 0.04 lies in the high range of commercially available paper-grade cationic starches $[2,39]$. The transesterification performed in a BM could be a feasible approach for the cost-effective synthesis of these lowly substituted cationic starch esters, even at a large scale, thanks to a low reaction time and the ease of product recovery.

The ${ }^{1} \mathrm{H}-\mathrm{NMR}$ and ATR-FTIR spectra of $\mathrm{SB}_{\mathrm{D}}$ were qualitatively identical to those of $\mathrm{SB}_{\mathrm{S}}$ synthesised after alkaline activation, i.e., those shown in Fig. 2 and $\underline{3 b}$. Examples are shown in the Supplementary Information (Fig. S2 and S3). The thermal degradation behaviour of $\mathrm{SB}_{\mathrm{D}}$ was found to be very similar to that of native starch, even when it was produced from cooked starch (Fig. S4). 
It can be suggested that the solvent-free synthesis, followed by dissolution in water and precipitation from ethanol, facilitated the recovery of a certain degree of molecular order.

\subsection{Degradation of starch}

Although both DMF and DMSO are, by themselves, non-derivatizing solvents for starch, the systems resulting from their combination with $\mathrm{MeBetCl}$ and alkali starch or sulfuric acid produced more solvolysis than expected.

Fig. 5 presents the values of inherent viscosities $\left(v_{\text {inh }}\right)$ for cooked starch, alkali starch and starch betainates obtained with a molar ratio of 2 (Experiments 2, 6, and 10 in Table 1; Experiments 2 and 4 in Table 2). Since measurements were performed with very dilute solutions, the inherent viscosity is roughly the same as the intrinsic viscosity. However, the molecular weight cannot be reliably estimated as the degree of branching of amylopectin in samples, on which the Mark-Houwink parameters are greatly dependent [40], is unknown.

In any case, valuable conclusions, even if these are qualitative remarks, can be highlighted. The lower viscosity of the starch alkoxide is not necessarily due to degradation in boiling $\mathrm{NaOH} / \mathrm{ethanol}$, but instead to conformational changes as charges along the polymer repel each other [41]. Of all SB samples, only the ones produced by BM had DP values in the range of the starting cooked starch. As an explanation for the low DS values found in DMSO, degradation in this solvent was more severe that in DMF. Furthermore, acidic media consistently caused more depolymerization than basic media. In fact, the value corresponding to $\mathrm{DMSO} / \mathrm{H}_{2} \mathrm{SO}_{4}$ is not given because no reliable measurement could be performed by following the same procedure, since starch had been broken down to small dextrins. The resulting maltodextrin betainates could be useful for certain applications, but a different separation method should be used to produce them with high yield. 


\subsection{Morphology of starch derivatives}

Images of starch, cooked starch and cationic starch derivatives are displayed in Fig. 6 and Fig. 7. While the former shows the shape of suspended particles in water with a pinch of iodine, the latter presents a SEM view of dry samples. Particles of the native starch used in this work (Fig. 6a and 7a) include spherical granules (diameter 1-10 $\mu \mathrm{m}$ ), lenticular granules and small aggregates. This granular or semicrystalline structure was lost in all treatments and not recovered in cold water (Fig. 6b-d). Hence, it could be said that there was hardly any retrogradation, which is desired in applications in which keeping the amorphous form is preferred, such as the food industry [25]. The $\mathrm{SB}_{\mathrm{D}}$ sample, in contrast, clearly recovered certain degree of molecular order (Fig. 7f), showing structures more similar to that of native starch.

The particle size of amorphous starch derivatives is not relevant, as the macromolecules simply agglomerated when precipitated from ethanol. However, the surface changes on these massive agglomerates are appreciable and meaningful. The smooth surface of cooked starch (Fig. 7b) was turned into rough and meso- or macroporous structures by transesterification (Fig. 7c-f), but not by conventional etherification towards highly-substituted CS (Fig. 7g). Even the dextrins formed in acidic DMSO were recovered as porous blocks (Fig. 7e).

When suspended in water, agglomerates of $\mathrm{SB}_{\mathrm{S}}$ with high DS (Experiment 2 in Table 1, Fig. 6c) underwent quick dispersion and even dissolution in cold water, unlike cooked starch (Fig. 6b) and lowly-substituted $\mathrm{SB}_{\mathrm{D}}$ (Fig. 6d). This enhancement of solubility is desired for some applications,

Eliminado: Fig. 8

Eliminado: Fig. 9

Eliminado: Fig. 8

Eliminado: $9 \mathrm{a}$

Eliminado: Fig. 8

Eliminado: Fig. 9

Eliminado: Fig. 9

Eliminado: Fig. 9

Eliminado: Fig. 9

Eliminado: Fig. 9

Eliminado: Fig. 8

Eliminado: Fig. 8

Eliminado: Fig. 8 such as to replace synthetic polyelectrolytes [42], but undesired for others, as commercially available cationic starches for paper sizing operations are generally insoluble

Micrographs at other scales can be found in the Supplementary Information (Fig. S5). 


\section{Conclusions}

Starch betainate, a more labile alternative to cationic starch ethers, was synthesised by

transesterification in two unprecedented ways. A key advantage of both procedures, namely reaction

in polar aprotic solvents and ball milling, lies in the high purity of the product. It may be suggested

that transesterification prevents the selectivity issues of the conventional etherification with

CHPTAC or EPTAC. In those processes, the introduction of another secondary hydroxyl group is

the source of different by-products. $\underline{\text { Clearly, this avoidance of an additional reactive group is shared }}$

with other possible routes towards starch betainates but, unlike esterification with the acyl chloride

of betaine, transesterification did not cause colouring when reaching high substitution.

\section{The success of the basic transesterification in $\mathrm{DMF}$, which achieved the highest degree of}

substitution (0.4), could be explained by an effective dissociation of alkali starch, while not

suffering from extensive solvolysis. Although there was solvolysis to a certain extent, shown by a

clear diminishment in intrinsic viscosity, this degradation was not as severe as that observed in

DMSO, which resulted in maltodextrins. Ball milling, in contrast, allowed starch betainate to retain a similar molecular weight and caused no appreciable degradation, particularly when alkaline activation was chosen.

\section{Acknowledgements}

This work was carried out under the Project inpactus -innovative products and technologies from eucalyptus, Project N. 21874 funded by Portugal 2020 through European Regional Development Fund (ERDF) in the frame of COMPETE 2020 n²46/AXIS II/2017. Authors would like to thank the Coimbra Chemical Centre, which is supported by the Fundação para a Ciência e a Tecnologia (FCT), through the projects UID/QUI/00313/2020 and COMPETE. Authors would also like to thank the CIEPQPF - Strategic Research Centre Project UIDB/00102/2020, funded by the Fundação para a
Eliminado: that are unprecedented in the literature

Eliminado: One of them involved long reactions $(24 \mathrm{~h})$ at $70{ }^{\circ} \mathrm{C}$ in a polar aprotic solvent: DMSO or, preferably, DMF. The other method was a solvent-free process that took place in a ball mill for 2 $\mathrm{h}$, without external heating and a frequency of $25 \mathrm{~s}^{-1}$.

Eliminado: obtained

Eliminado:

Eliminado: among other possible routes towards starch betainates, Eliminado: along with the reaction being generally carried out in strongly alkaline media,

Eliminado: In contrast, no by-products were detected in SB samples.

Eliminado: The highest degree of substitution, 0.4 , was obtained by depolymerizing the starch with amylase, pre-activating it in $\mathrm{NaOH} /$ ethanol and making it react in DMF with three moles of methyl betainate chloride per mole of anhydroglucose unit.

Eliminado: While the degrees of substitution attained $(0.04)$ were lower than those achieved in DMF, they are still in the high range of paper-grade cationic starches. 
Ciência e Tecnologia (FCT). M.S. acknowledges the PhD grant BDE 03|POCI-01-0247-FEDER021874. R.A. acknowledges the post-doc grant BPD 02|POCI-01-0247-FEDER-021874.

\section{References}

[1] V. Haack, T. Heinze, G. Oelmeyer, W.M. Kulicke, Starch derivatives of high degree of functionalization, 8 synthesis and flocculation behavior of cationic starch polyelectrolytes, Macromol. Mater. Eng. 287 (2002) $495-$ 502.

[2] M. Sharma, R. Aguado, D. Murtinho, A.J.M. Valente, A.P. Mendes De Sousa, P.J.T. Ferreira, A review on cationic starch and nanocellulose as paper coating components, Int. J. Biol. Macromol. 162 (2020) $\underline{578-598}$. https://doi.org/10.1016/j.ijbiomac.2020.06.131.

[3] J. Liu, R. Yang, F. Yang, Effect of the starch source on the performance of cationic starches having similar degree of substitution for papermaking using deinked pulp, BioResources. 10 (2015) 922-931.

[4] H. Li, Y. Qi, Y. Zhao, J. Chi, S. Cheng, Starch and its derivatives for paper coatings: A review, Prog. Org. Coatings. 135 (2019) 213-227. https://doi.org/10.1016/j.porgcoat.2019.05.015.

[5] S. Pal, D. Mal, R.P. Singh, Cationic starch: An effective flocculating agent, Carbohydr. Polym. 59 (2005) 417423.

[6] H. Zhou, L. Zhou, X. Yang, Optimization of preparing a high yield and high cationic degree starch graft copolymer as environmentally friendly flocculant: Through response surface methodology, Int. J. Biol. Macromol. 118 (2018) 1431-1437.

[7] M.J. Farrell, P.J. Hauser, Cationic cotton, reservations to reality, AATCC Rev. 13 (2013) 56-63.

[8] J. Bendoraitiene, E. Lekniute-Kyzike, R. Rutkaite, Biodegradation of cross-linked and cationic starches, Int. J. Biol. Macromol. 119 (2018) 345-351.

[9] P. Maćczak, H. Kaczmarek, M. Ziegler-Borowska, Recent achievements in polymer bio-based flocculants for water treatment, Materials (Basel). 13 (2020). https://doi.org/10.3390/ma13183951.

[10] N. Masina, Y.E. Choonara, P. Kumar, L.C. Toit, M. Govender, S. Indermun, V. Pillay, A review of the chemical modification techniques of starch, Carbohydr. Polym. 157 (2017) 1226-1236.

[11] R. Auzély-Velty, M. Rinaudo, Synthesis of starch derivatives with labile cationic groups, Int. J. Biol. Macromol. 31 (2003) 123-129.

[12] H. Granö, J. Yli-Kauhaluoma, T. Suortti, J. Käki, K. Nurmi, Preparation of starch betainate: a novel cationic starch derivative, Carbohydr. Polym. (2000). https://doi.org/10.1016/S0144-8617(99)00146-0.

[13] I. Palej Jakopović, S. Kapić, S. Alihodžić, V. Šunjić, Ethers from esters; From exceptional transformation to 
synthetic method, Arkivoc. (2015) 300-326. https://doi.org/10.3998/ark.5550190.p008.960.

[14] A. Laine, H. Luttikhedde, A process for the preparation of hydroxy polymer esters and their use, EP2079762, 2012.

[15] S. Khodaverdian, B. Dabirmanesh, A. Heydari, E. Dashtban-moghadam, K. Khajeh, F. Ghazi, Activity, stability and structure of laccase in betaine based natural deep eutectic solvents, Int. J. Biol. Macromol. 107 (2018) 2574 2579. https://doi.org/10.1016/j.ijbiomac.2017.10.144.

[16] S. Huang, Q. Ma, Q. Hou, T. Zuo, Z. Zhang, W. Ni, Identification and quantitative chemical analysis of betaines in different organic wastes and their bioconversion composts, Bioresour. Technol. 328 (2021) 124857. https://doi.org/10.1016/j.biortech.2021.124857.

[17] H. Winkler, W. Vorwerg, H. Wetzel, Synthesis and properties of fatty acid starch esters, Carbohydr. Polym. 98 (2013) 208-216. https://doi.org/10.1016/j.carbpol.2013.05.086.

[18] R. Dicke, D.O. Klemm, Synthesis of regioselectively functionalized polysaccharide esters avoiding protecting groups, in: Am. Chem. Soc. Polym. Prepr. Div. Polym. Chem., 2000.

[19] A. Schenzel, A. Hufendiek, C. Barner-Kowollik, M.A.R. Meier, Catalytic transesterification of cellulose in ionic liquids: Sustainable access to cellulose esters, Green Chem. 16 (2014) 3266-3271. https://doi.org/10.1039/c4gc00312h.

[20] Z. Söyler, M.A.R. Meier, Catalytic Transesterification of Starch with Plant Oils: A Sustainable and Efficient Route to Fatty Acid Starch Esters, ChemSusChem. 10 (2017) 182-188. https://doi.org/10.1002/cssc.201601215.

[21] T.A. Wani, F.A. Masoodi, R. Akhter, F.A. Sofi, Techno-functional characterization of chitosan nanoparticles prepared through planetary ball milling, Int. J. Biol. Macromol. 154 (2020) 166-172. https://doi.org/10.1016/J.IJBIOMAC.2020.03.034.

[22] L. Dai, C. Li, J. Zhang, F. Cheng, Preparation and characterization of starch nanocrystals combining ball milling with acid hydrolysis, Carbohydr. Polym. 180 (2018) 122-127. https://doi.org/10.1016/J.CARBPOL.2017.10.015.

[23] Z.-Q. Huang, X. Xie, Y. Chen, J. Lu, Z.-F. Tong, Ball-milling treatment effect on physicochemical properties and features for cassava and maize starches, Comptes Rendus Chim. 11 (2008) 73-79. https://doi.org/10.1016/J.CRCI.2007.04.008.

[24] Y.J. Kim, T. Suzuki, T. Hagiwara, I. Yamaji, R. Takai, Enthalpy relaxation and glass to rubber transition of amorphous potato starch formed by ball-milling, Carbohydr. Polym. 46 (2001) 1-6. https://doi.org/10.1016/S0144-8617(00)00274-5.

[25] T.Y. Liu, Y. Ma, S.F. Yu, J. Shi, S. Xue, The effect of ball milling treatment on structure and porosity of maize 
starch granule, Innov. Food Sci. Emerg. Technol. 12 (2011) 586-593.

https://doi.org/10.1016/J.IFSET.2011.06.009.

[26] X. Tan, B. Zhang, L. Chen, X. Li, L. Li, F. Xie, Effect of planetary ball-milling on multi-scale structures and pasting properties of waxy and high-amylose cornstarches, Innov. Food Sci. Emerg. Technol. 30 (2015) 198207. https://doi.org/10.1016/J.IFSET.2015.03.013.

[27] M. Ahmad, A. Gani, F.A. Masoodi, S.H. Rizvi, Influence of ball milling on the production of starch nanoparticles and its effect on structural, thermal and functional properties, Int. J. Biol. Macromol. 151 (2020) 85-91. https://doi.org/10.1016/J.IJBIOMAC.2020.02.139.

[28] Y. Su, H. Du, Y. Huo, Y. Xu, J. Wang, L. Wang, S. Zhao, S. Xiong, Characterization of cationic starch flocculants synthesized by dry process with ball milling activating method, Int. J. Biol. Macromol. 87 (2016) $34-40$.

[29] N. Li, M. Niu, B. Zhang, S. Zhao, S. Xiong, F. Xie, Effects of concurrent ball milling and octenyl succinylation on structure and physicochemical properties of starch, Carbohydr. Polym. 155 (2017) 109-116. https://doi.org/10.1016/J.CARBPOL.2016.08.063.

[30] R. Owusu-Apenten, Introduction to food chemistry, 2004. https://doi.org/10.5860/choice.43-2809.

[31] R.G. Webb, M.W. Haskell, C.H. Stammer, A Nuclear Magnetic Resonance Method for Distinguishing a-Amino Acids from B and y isomers, J. Org. Chem. 34 (1969) 576-580.

[32] C.L. Øpstad, T.B. Melø, H.R. Sliwka, V. Partali, Formation of DMSO and DMF radicals with minute amounts of base, Tetrahedron. 65 (2009) 7616-7619. https://doi.org/10.1016/j.tet.2009.06.109.

[33] X.F. Wu, K. Natte, The Applications of Dimethyl Sulfoxide as Reagent in Organic Synthesis, Adv. Synth. Catal. $\underline{338}$ (2016) 336-352. https://doi.org/10.1002/adsc.201501007.

[34] H. Tang, B.P. Hills, Use of 13C MAS NMR to study domain structure and dynamics of polysaccharides in the native starch granules, Biomacromolecules. 4 (2003) 1269-1276. https://doi.org/10.1021/bm0340772.

[35] J.J. Cael, J.L. Koenig, J. Blackwell, Infrared and raman spectroscopy of carbohydrates, Carbohydr. Res. 19 (1974) 297-310. https://doi.org/10.1016/s0008-6215(00)82465-9.

[36] T. Yue, X. Wu, Depressing iron mineral by metallic-starch complex (MSC) in reverse flotation and its mechanism, Minerals. (2018). https://doi.org/10.3390/min8030085.

[37] A. Hebeish, A. Higazy, A. El-Shafei, S. Sharaf, Synthesis of carboxymethyl cellulose (CMC) and starch-based hybrids and their applications in flocculation and sizing, Carbohydr. Polym. 79 (2010) 60-69.

[38] P. Wang, X. Wu, X. Dong-hua, X. Kun, T. Ying, D. Xi-bing, L. Wen-bo, Preparation and characterization of cationic corn starch with a high degree of substitution in dioxane - THF - water media, Carbohydr. Res. 344 


\section{(2009) 851-855.}

[39] K. Niegelhell, A. Chemelli, J. Hobisch, T. Griesser, H. Reiter, U. Hirn, S. Spirk, Interaction of industrially relevant cationic starches with cellulose, Carbohydr. Polym. 179 (2018) 290-296.

[40] M.M. Millard, F.R. Dintzis, J.L. Willett, J.A. Klavons, Light-scattering molecular weights and intrinsic viscosities of processed waxy maize starches in 90\% dimethyl sulfoxide and H2O, Cereal Chem. 74 (1997) $687-$ 691. https://doi.org/10.1094/CCHEM.1997.74.5.687.

[41] R. Aguado, A.F. Lourenço, P.J.T. Ferreira, A. Moral, A. Tijero, The relevance of the pretreatment on the chemical modification of cellulosic fibers, Cellulose. 26 (2019) 5925-5936. https://doi.org/10.1007/s10570-01902517-7.

[42] R. Aguado, A.F. Lourenço, P.J. Ferreira, A. Moral, A. Tijero, Cationic cellulosic derivatives as flocculants in papermaking, Cellulose. 24 (2017) 3015-3027. https://doi.org/10.1007/s10570-017-1313-y. 
Table 1. Reaction conditions and degree of substitution for the transesterification of starch in solution.

\begin{tabular}{|c|c|c|c|c|c|}
\hline Exp. & $\begin{array}{l}\text { Type } \\
\text { of } \\
\text { starch }\end{array}$ & Medium & $\begin{array}{l}\text { MeBetCl/ } \\
\text { AGU }\end{array}$ & Solvent & DS \\
\hline 1 & Cooked & \multirow{4}{*}{ Alkaline } & 1 & DMF & 0.16 \\
\hline 2 & Cooked & & 2 & DMF & 0.33 \\
\hline 3 & Cooked & & 3 & DMF & 0.40 \\
\hline 4 & Native & & 2 & DMF & 0.01 \\
\hline 5 & Cooked & \multirow{4}{*}{ Acidic } & 1 & DMF & 0.08 \\
\hline 6 & Cooked & & 2 & DMF & 0.14 \\
\hline 7 & Cooked & & 3 & DMF & 0.18 \\
\hline 8 & Native & & 2 & DMF & 0.006 \\
\hline$\underline{9}$ & Cooked & \multirow{4}{*}{$\underline{\text { Alkaline }}$} & $\underline{1}$ & $\underline{\text { DMSO }}$ & $\underline{0.01}$ \\
\hline$\underline{10}$ & Cooked & & $\underline{2}$ & $\underline{\mathrm{DMSO}}$ & $\underline{0.05}$ \\
\hline$\underline{11}$ & Cooked & & $\underline{3}$ & $\underline{\mathrm{DMSO}}$ & $\underline{0.02}$ \\
\hline$\underline{12}$ & Native & & $\underline{2}$ & $\underline{\mathrm{DMSO}}$ & $\underline{0.07}$ \\
\hline$\underline{13}$ & Cooked & \multirow{4}{*}{ Acidic } & $\underline{1}$ & $\underline{\mathrm{DMSO}}$ & $\underline{0.01}$ \\
\hline$\underline{14}$ & Cooked & & $\underline{2}$ & $\underline{\mathrm{DMSO}}$ & $\underline{0.10}$ \\
\hline$\underline{15}$ & $\underline{\text { Cooked }}$ & & $\underline{3}$ & $\underline{\mathrm{DMSO}}$ & $\underline{0.11}$ \\
\hline 16 & Native & & $\underline{2}$ & $\underline{\mathrm{DMSO}}$ & $\underline{0.01}$ \\
\hline
\end{tabular}

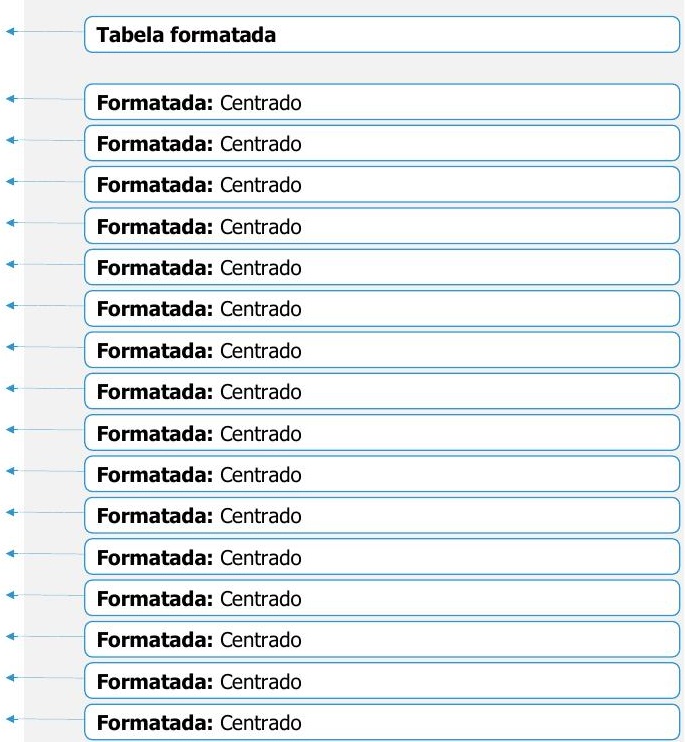


Table 2. Reaction conditions and DS achieved in solid-state transesterification.

\begin{tabular}{llll} 
Exp. & Medium & $\begin{array}{l}\text { eq. } \\
\text { MeBetCl }\end{array}$ & DS \\
\hline 1 & Alkaline & 1 & 0.006 \\
2 & 2 & 0.04 \\
3 & Acidic & 1 & 0.006 \\
4 & & 2 & 0.04 \\
\hline
\end{tabular}




\section{Figure / scheme captions — in order of appearance along the text}

Figure 1. Simplified diagram of the experimental plan to synthesise starch betainate.

Scheme 1. Esterification (reaction A) of betaine hydrochloride.

Figure 2. ${ }^{1} \mathrm{H}-\mathrm{NMR}$ spectra of: a) $\mathrm{MeBetCl}$ (DMSO-d6); b) starch $\left(\mathrm{D}_{2} \mathrm{O}\right.$ ); c) SB obtained in alkaline medium, Experiment 2 in Table $1\left(\mathrm{D}_{2} \mathrm{O}\right)$. HDO signal has been cut.

Figure 3. ATR-FTIR spectra for: a) BetH (bottom) and $\mathrm{MeBetCl}$ (top); b) SB obtained in alkaline and acidic media (MeBetCl/AGU =2, DMF) compared to those of cooked and alkali cooked starches.

Scheme 2. Transesterification reactions of $\mathrm{MeBetCl}$ and starch to yield SB. It should be noted that substitution on carbons 2 and 3 of AGU is also possible.

Figure 4 . TGA (a) and DTG (b) curves for highly substituted starch betainates, obtained in either alkaline $(\mathrm{MeBetCl} / \mathrm{AGU}=2, \mathrm{DS}=0.33)$ and acidic media $(\mathrm{MeBetCl} / \mathrm{AGU}=2, \mathrm{DS}=0.14)$, compared to those curves for native starch, cooked starch, and a conventional cationic starch ether (DS $=0.3$ ).

Figure 5. Inherent viscosity $\left(v_{\mathrm{inh}}\right)$ of starch derivatives. For all SB samples, the MeBetCl/AGU ratio was 2.

Figure 6. Optical micrographs of native corn starch (a), cooked starch (b), SB produced in basic DMF (c), and SB obtained by solid-state transesterification (d).

Figure 7. Scanning electron micrographs of dry native starch (a); cooked starch (b); betainates thereof that were synthesised in basic DMF (c), acidic DMF (d), acidic DMSO (e) and BM after alkaline activation (f); $\mathrm{CS}$ ether $(\mathrm{g})$.

Eliminado:

Eliminado: ) and ${ }^{13} \mathrm{C}-\mathrm{NMR}$ (b) spectra of

Formatada: Inferior à linha

Formatada: Inferior à linha

Eliminado: Figure $4 .{ }^{1} \mathrm{H}$ NMR spectrum for SB obtained in alkaline medium (DS $=0.33$; Experiment 2 in Table 1) compared to that of starch $\left(\mathrm{D}_{2} \mathrm{O}\right)$. HDO signal $(4.8 \mathrm{ppm})$ has been cut.II

Figure 5. ATR-FTIR spectra of SB obtained in alkaline and acidic media $(\mathrm{MeBetCl} / \mathrm{AGU}=2, \mathrm{DMF})$ compared to those of cooked and alkali cooked starches.

Eliminado: 6

Eliminado: 7

Eliminado: 8

Eliminado: 9 


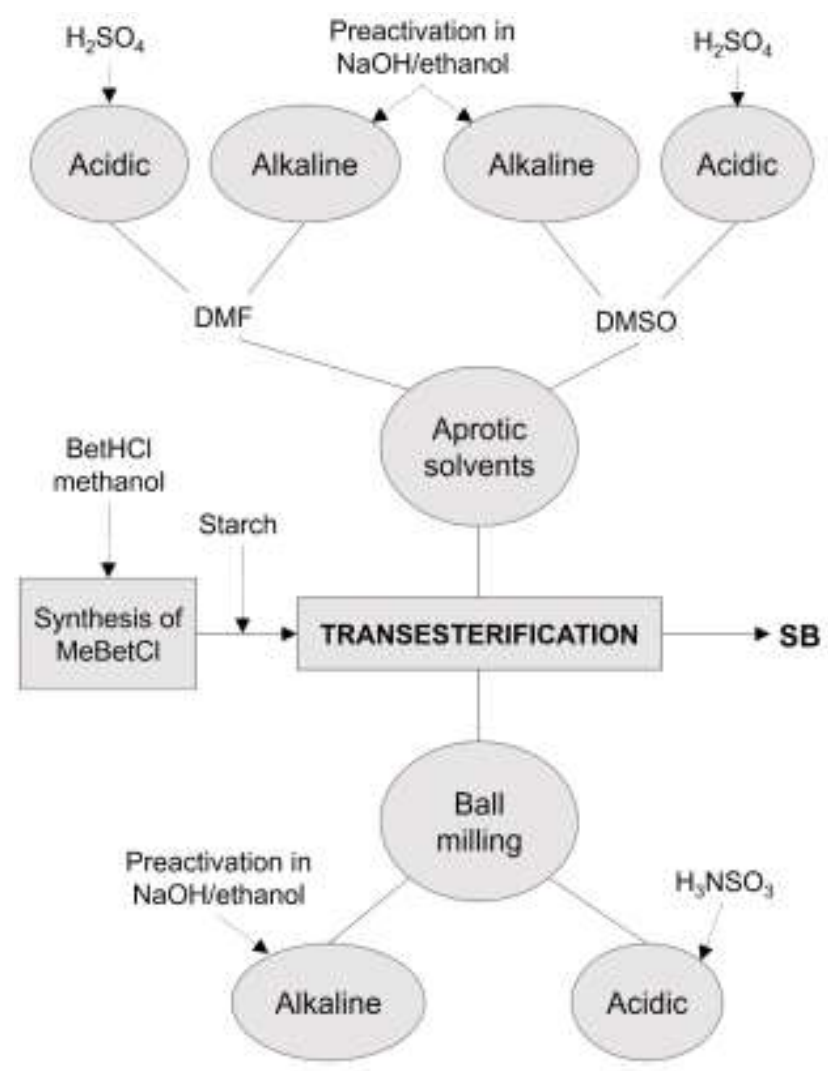

Figure 1. Simplified diagram of the experimental plan to synthesise starch betainate.

For International Journal of Biological Macromolecules (Sharma, Aguado, Murtinho, Valente, Ferreira, 2021) 


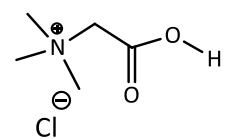

Betaine hydrochloride

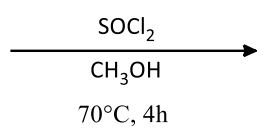

A<smiles>COC(=O)C[N+](C)(C)Cl</smiles>

Methyl betainate

chloride

Scheme 3 , Esterification (reaction A) of betaine hydrochloride.

Eliminado:

For International Journal of Biological Macromolecules (Sharma, Aguado, Murtinho, Valente, Ferreira, 2021) 


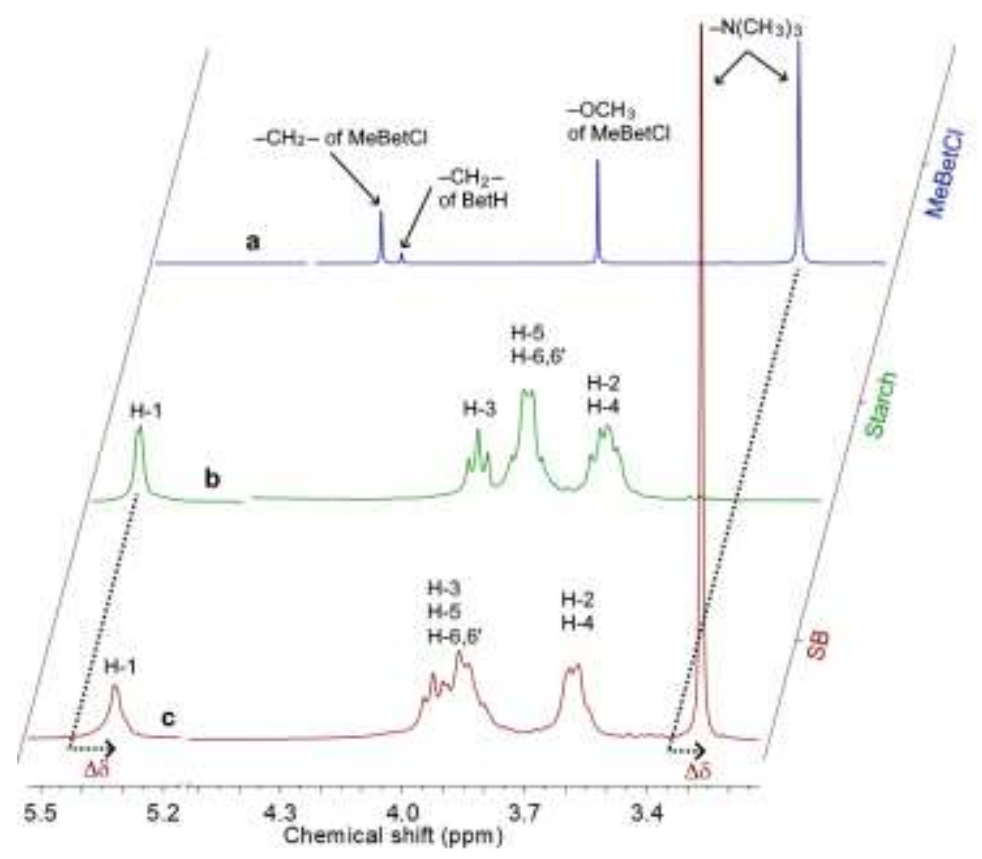

Figure 2. ${ }^{1} \mathrm{H}-\mathrm{NMR}$ spectra of: a) $\mathrm{MeBetCl}$ (DMSO-d6); b) starch $\left(\mathrm{D}_{2} \mathrm{O}\right)$; c) SB obtained in alkaline medium, Experiment 2 in Table $1\left(\mathrm{D}_{2} \mathrm{O}\right)$. HDO signal has been cut,

For International Journal of Biological Macromolecules (Sharma, Aguado, Murtinho, Valente, Ferreira, 2021) a

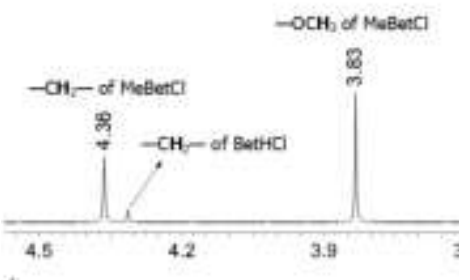

b

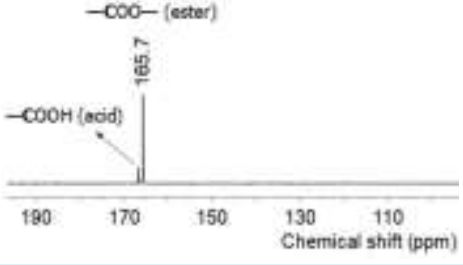

Eliminado:

Formatada: Português (Portugal)

Eliminado: ${ }^{1} \mathrm{H}-\mathrm{NMR}$ (a) and ${ }^{13} \mathrm{C}-\mathrm{NMR}$ (b) spectra of MeBetC (DMSO-d6)

Eliminado: 

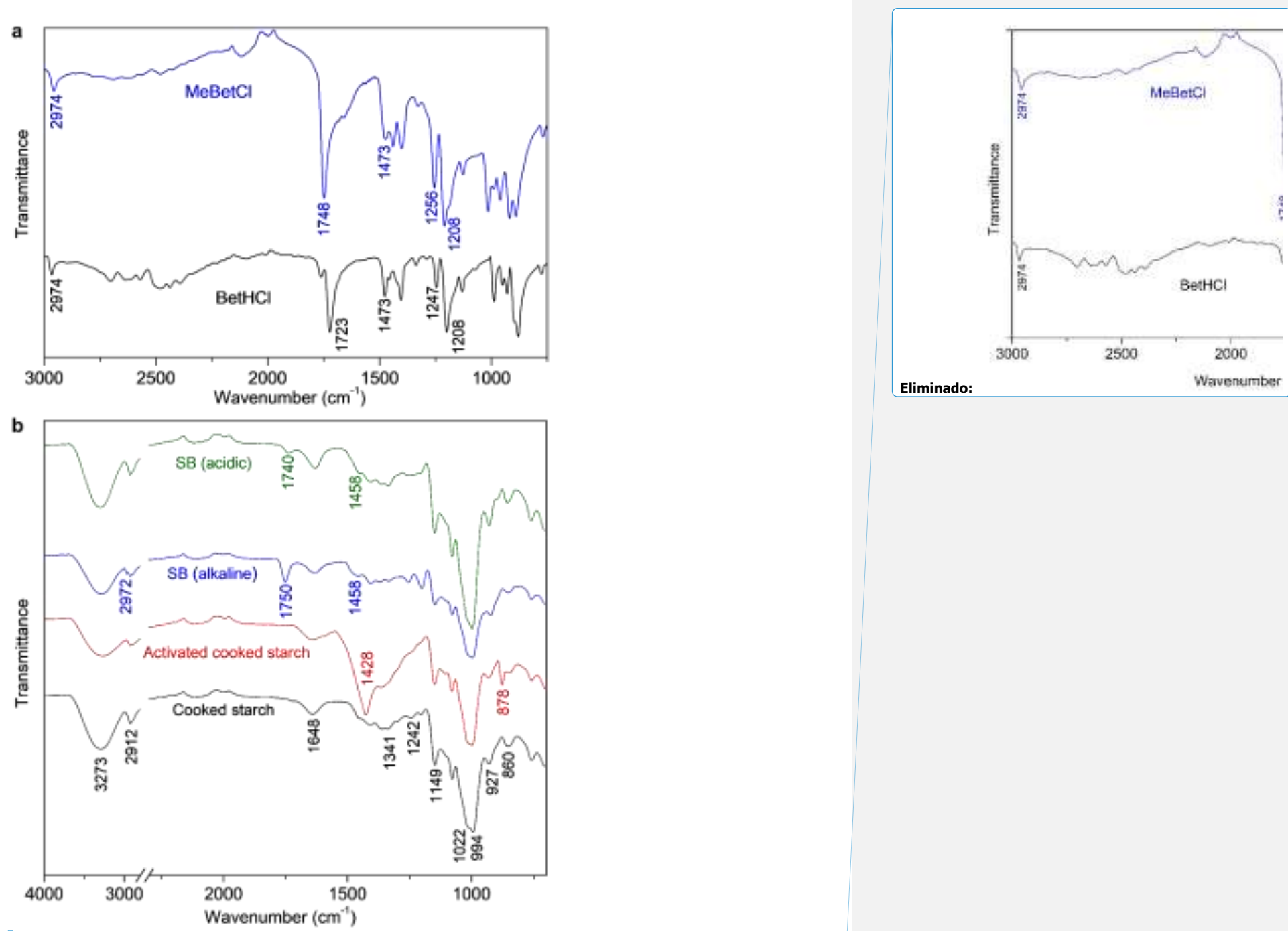

Figure 3. Figure 3. ATR-FTIR spectra for: a) BetH (bottom) and $\mathrm{MeBetCl}$ (top); b) SB obtained in alkaline and acidic media (MeBetCl/AGU =2, DMF) compared to those of cooked and alkali cooked starches.

For International Journal of Biological Macromolecules (Sharma, Aguado, Murtinho, Valente, Ferreira, 2021)

Formatada: Rasurado

Eliminado: ATR-FTIR spectra for BetH (bottom) and MeBetC (top). .I 


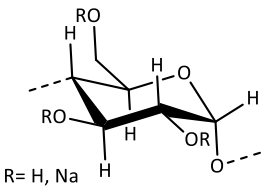

Alkalized anhydroglucose unit

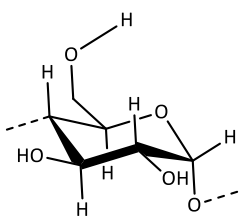<smiles>COC(=O)C[N+](C)(C)Cl</smiles>

Anhydroglucose unit
$+\overbrace{\mathrm{Cl}}^{\stackrel{\oplus}{\ominus}} \overbrace{\mathrm{O}}^{\mathrm{OCH}_{3}}$

Methyl betainate chloride

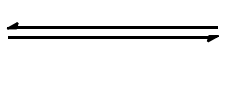

B

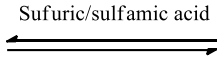

C

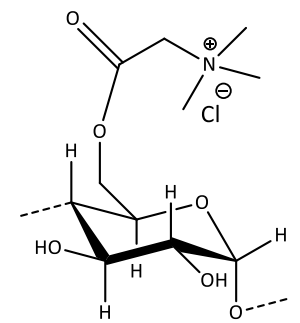

Starch betainate chloride

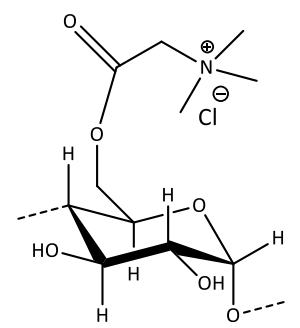

Starch betainate chloride

Scheme 4, Transesterification reactions of $\mathrm{MeBetCl}$ and starch to yield $\mathrm{SB}$. It should be noted that substitution on carbons 2 and 3 of AGU is also possible.

For International Journal of Biological Macromolecules (Sharma, Aguado, Murtinho, Valente, Ferreira, 2021)

$$
\text { chloride }
$$

Formatada: Normal, Não manter com o seguinte Eliminado: II 

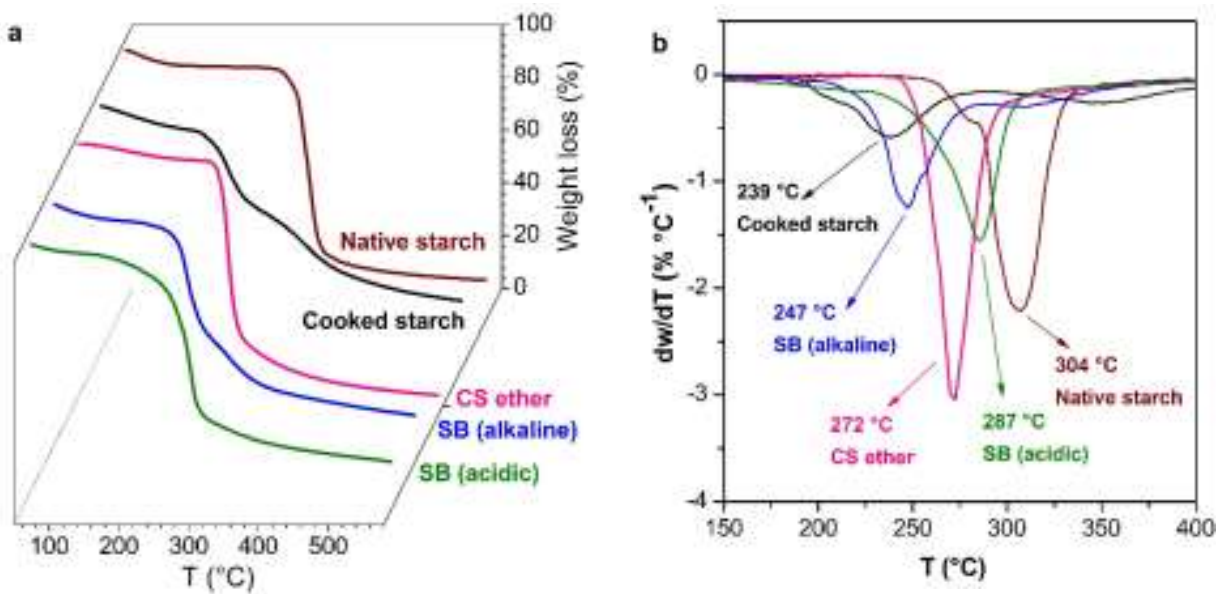

Figure 4. TGA (a) and DTG (b) curves for highly substituted starch betainates, obtained in either alkaline $(\mathrm{MeBetCl} / \mathrm{AGU}=2, \mathrm{DS}=0.33)$ and acidic media $(\mathrm{MeBetCl} / \mathrm{AGU}=2, \mathrm{DS}=0.14)$, compared to those curves for native starch, cooked starch, and a conventional cationic starch ether ( $\mathrm{DS}=0.3$ ).

For International Journal of Biological Macromolecules (Sharma, Aguado, Murtinho, Valente, Ferreira, 2021)

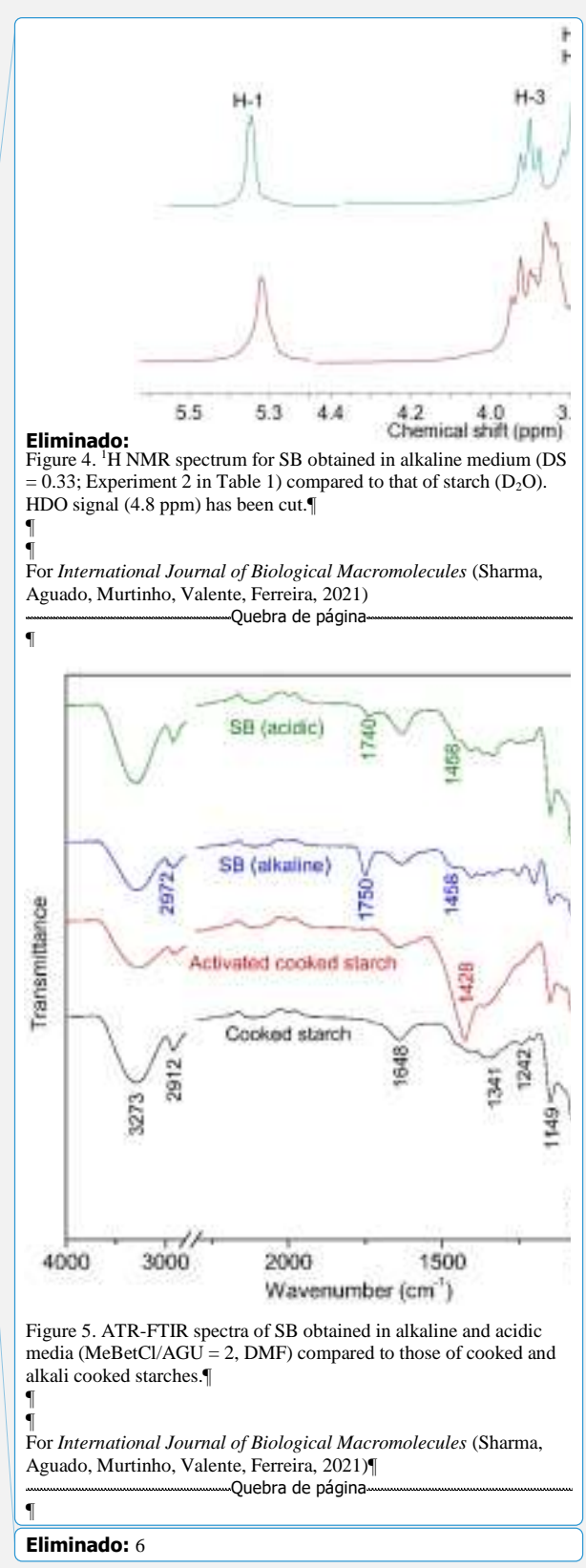




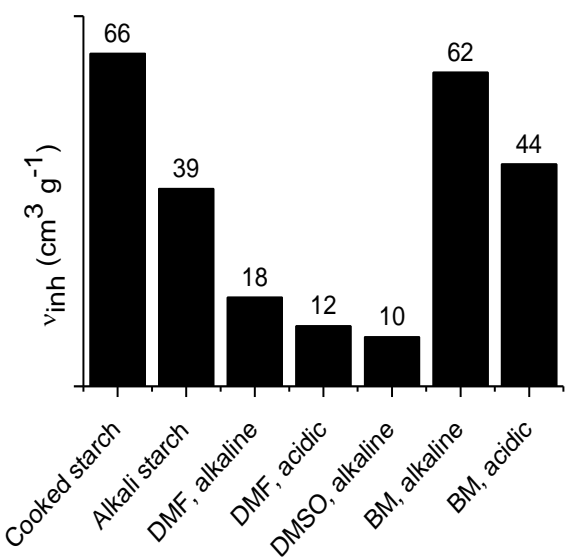

Figure 5. Inherent viscosity $\left(y_{\text {inh }}\right)$ of starch derivatives. For all SB samples, the MeBetCl/AGU ratio was 2.

For International Journal of Biological Macromolecules (Sharma, Aguado, Murtinho, Valente, Ferreira, 2021) 


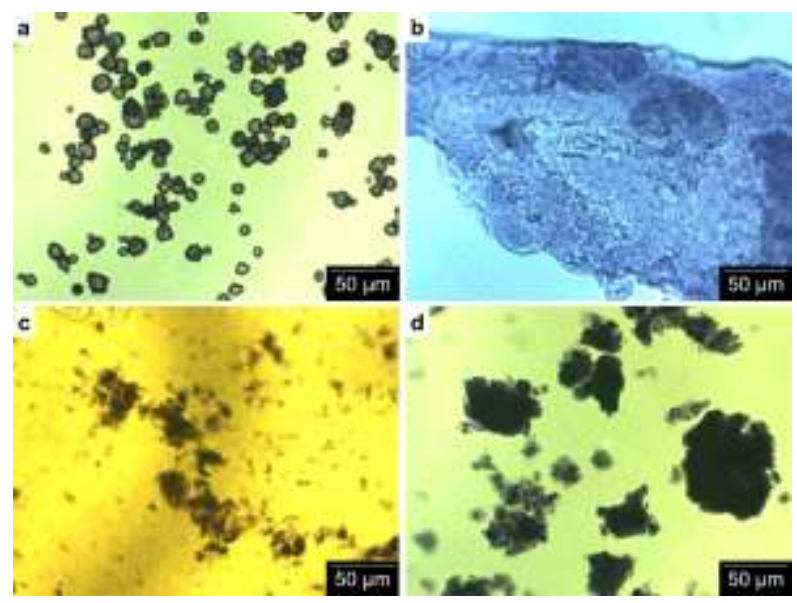

Figure 6. Optical micrographs of native corn starch (a), cooked starch (b), SB produced in basic DMF (c), and SB obtained by solid-state transesterification (d).

For International Journal of Biological Macromolecules (Sharma, Aguado, Murtinho, Valente, Ferreira, 2021) 

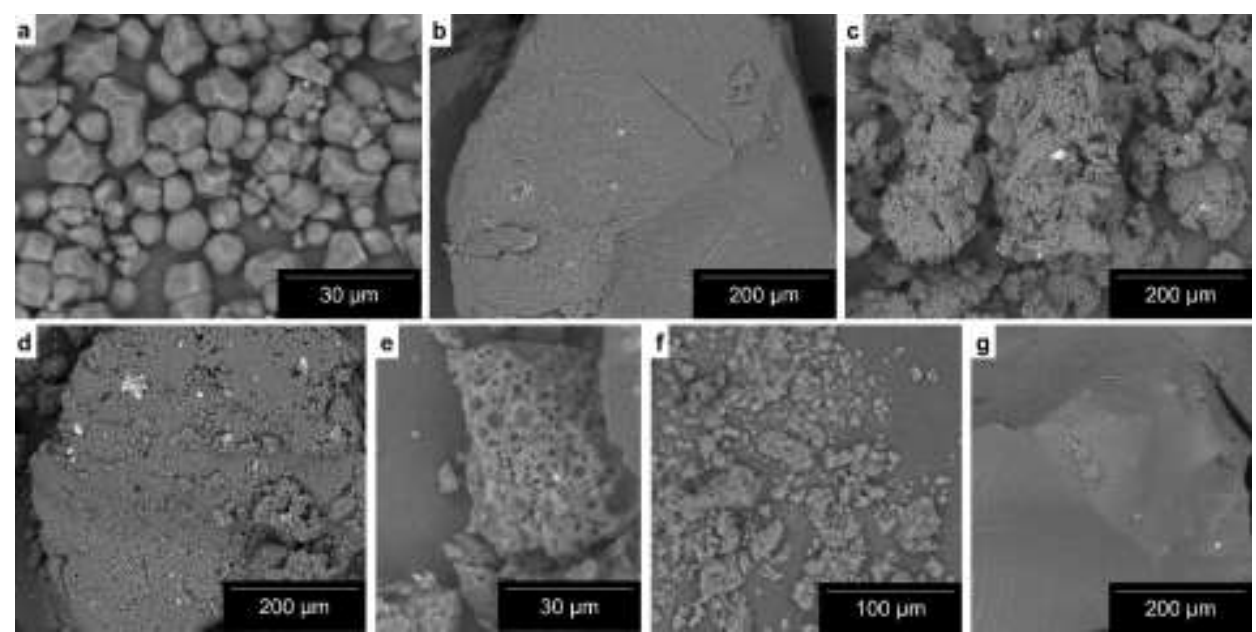

Figure 7. Scanning electron micrographs of dry native starch (a); cooked starch (b); betainates thereof that were synthesised in basic DMF (c), acidic DMF (d), acidic DMSO (e) and BM after alkaline activation (f); CS ether $(\mathrm{g})$.

For International Journal of Biological Macromolecules (Sharma, Aguado, Murtinho, Valente, Ferreira, 2021) 